\title{
ESA Parabolic Flights, Drop Tower and Centrifuge Opportunities for University Students
}

\author{
Natacha Callens - Javier Ventura-Traveset - Thomas-Louis de Lophem • \\ Carlos Lopez de Echazarreta • Vladimir Pletser • Jack J. W. A. van Loon
}

Received: 29 October 2009 / Accepted: 10 February 2010 / Published online: 23 February 2010

(C) The Author(s) 2010. This article is published with open access at Springerlink.com

\begin{abstract}
Fly Your Thesis!-An Astronaut Experience" is an educational programme launched by the ESA Education Office that aims to offer to European students the unique opportunity to design, build, and eventually fly, a scientific experiment as part of their Master or Ph.D. thesis. Selected teams accompany their experiments onboard the Zero-G aircraft for a series of three flights, each consisting of 30 parabolas, with each parabola providing about $20 \mathrm{~s}$ of microgravity. ESA Education Office financially supports the flights and part of the hardware development, as well as travel and accommodation for participants. For the first edition of this programme, four student teams were selected to participate in the 51st ESA Microgravity Research Campaign in November 2009. The 2010 edition of the programme was launched in April 2009 and the final selection was announced in January 2010. In parallel,
\end{abstract}

N. Callens $(\varangle) \cdot$ J. Ventura-Traveset · T.-L. de Lophem

C. Lopez de Echazarreta

European Space Agency (ESA), ESA-ESAC, LEX-COV,

P.O. Box 78, 28691 Villanueva de la Cañada, Madrid, Spain

e-mail: natacha.callens@esa.int

\section{Pletser}

European Space Agency (ESA), ESA-ESTEC, D/HSF-UPS,

P.O. Box 299, 2200 AG Noordwijk, The Netherlands

e-mail: Vladimir.Pletser@esa.int

J. J. W. A. van Loon

European Low Gravity Research Association (ELGRA),

Dutch Experiment Support Center (DESC),

ACTA, Dept. Oral Cell Biology, VU-University,

van der Boechorststraat 7,

1081 BT Amsterdam, The Netherlands

e-mail: j.vanloon@vumc.nl
ESA Education Office is setting up two new hands-on activities to provide European university students with access to drop tower (up to $9.3 \mathrm{~s}$ of microgravity) and centrifuge (from 1 to 20 times Earth's gravity) facilities. Through ELGRA, the selected student teams are working in close contact with renowned European scientists working in gravity-related research. This paper will introduce the three new educational programmes and present the selected experiments, as well as give information to students interested in the future editions.

Keywords Microgravity $\cdot$ Hypergravity $\cdot$ Parabolic flight $\cdot$ Drop Tower $\cdot$ Centrifuge $\cdot$ Education

\section{Abbreviations}

ESA

ELGRA

FYT

DYT

SYT

\section{Introduction}

The ESA Education Office was set up in 1998. Its purpose is to motivate young people to study science, engineering and technology subjects and to ensure a qualified workforce for ESA and the European space sector in the future. To this end the ESA Education Office is supporting several hands-on activities, including small student satellites and student experiments on sounding rockets, high altitude balloons and microgravity/hypergravity platforms (Emma et al. 2008). 
Parabolic flights were proposed by ESA to foster university students' interest in microgravity research all over Europe. The Student Parabolic Flight Campaign was the first hands-on activity offered by ESA, starting in 1994. The last full ESA student campaign was held in 2006. Thereafter, the whole programme was thoroughly reviewed and several managerial and safety recommendations were made for future campaigns. Following these recommendations and specific interactions between the ESA Education Office and the ESA Directorate of Human Space Flight, a new programme concept called "Fly Your Thesis!-An Astronaut Experience" (FYT) was agreed upon.

"Fly Your Thesis!” Programme

The FYT programme aims to offer European students the unique opportunity to design, build, and eventually fly a scientific experiment to be performed in microgravity. These experiments must be part of the students' Master or Ph.D. thesis.

Teams composed of two to four students and supported by an endorsing professor can apply, each year, by submitting a letter of intent to the ESA Educa- tion Office. A review board composed of experts from the European Low Gravity Research Association (ELGRA; http://www.elgra.org), the ESA Directorate of Human Space Flight and the ESA Education Office pre-selects up to 20 teams and invites them to develop more detailed scientific and technical proposals, with the support of an ELGRA scientific mentor. As a conclusion to this pre-selection phase, the student teams present their projects to the review board during a dedicated workshop held at an ESA centre.

After this workshop, up to four teams are selected to build and perform their experiment during an ESA Microgravity Research Campaign (Pletser et al. 2009). The students accompany their experiments onboard the A300 Zero-G aircraft, operated by the company Novespace http://www.novespace.fr) from Bordeaux in France, for a series of three flights of 30 parabolas each, with each parabola providing about $20 \mathrm{~s}$ of microgravity (see Fig. 1). During this campaign, students work in close contact with renowned European scientists carrying out their own research. The ESA Education Office financially supports the flights and part of the hardware development, as well as travel and accommodation for participants.

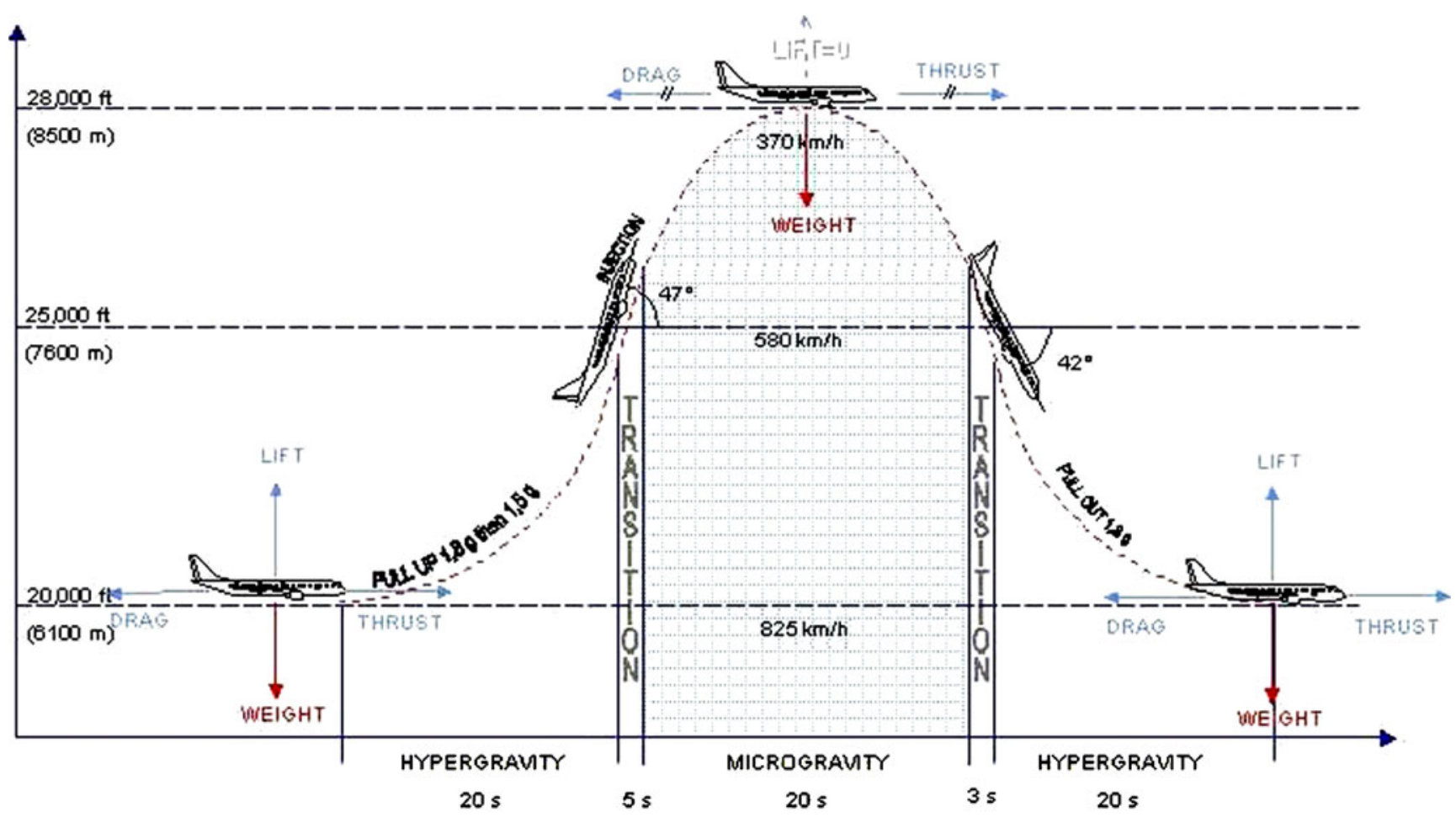

Fig. 1 Profile of the parabola performed by the Airbus A300 Zero-G during a parabolic flight (Credits Novespace). The parabola includes phases with different gravity levels $g$ : $1-2 g$

(while climbing), $0 g$ (about $20 \mathrm{~s}$ per parabola), $2 g$ (while going back to a horizontal trajectory); $1 g$ (on a horizontal flight) 
“Fly Your Thesis!” 2009

For the first version of the FYT programme, the review board selected four student teams who participated successfully in the 51st ESA Microgravity Research Campaign in November 2009 (Callens et al. 2009).

\section{The Complex Team}

The Complex team is composed of three Norwegian students from the Norwegian University of Science and Technology in Trondheim (Norway). They investigated the phenomenon of flow birefringence in samples containing synthetic clay particles suspended in water, in order to achieve a deeper understanding of the selforganisation of these nanoparticles. The microgravity environment eliminated problems related to sedimentation and convection that could have interfered with the pure Brownian dynamics of the system.

Flow birefringence is a phenomenon that occurs in colloid suspensions that contain anisotropic particles, such as clays particles. When the suspension flows, the particles align with the flow, in order to minimize particle velocities. This self-organisation induces a strong and colourful flow birefringence as illustrated in Fig. 2.

The idea of this student experiment was to compare the relaxation time of the birefringence of several samples placed in separate sample cells (Okubo et al. 2002). Prior to microgravity an identical flow was induced in all the samples. In microgravity, the flow was stopped and the decaying birefringence was recorded. By varying salt content and clay concentration, they studied the impact of these factors on the particleparticle interactions of the dispersed clay.

The setup consisted of three connected parts: a liquid circulation system, a sample observation system and an electrical system. Most of the components were mounted inside an aluminum box to contain the liquids and block unwanted light. The main instruments of their setup were glass sample cells, a LED based light source to illuminate the samples, a pump to induce a flow in the samples, two crossed polarisers placed on each side of the samples and a video camera that monitors the flow birefringence inside the sample cells (see Fig. 3).

The important role of flow and orientation of clay particles in the context of soil stability is not yet properly understood. The successful students' experiment may therefore provide, in the coming months, more insight on some of the basic processes affecting soil stability.

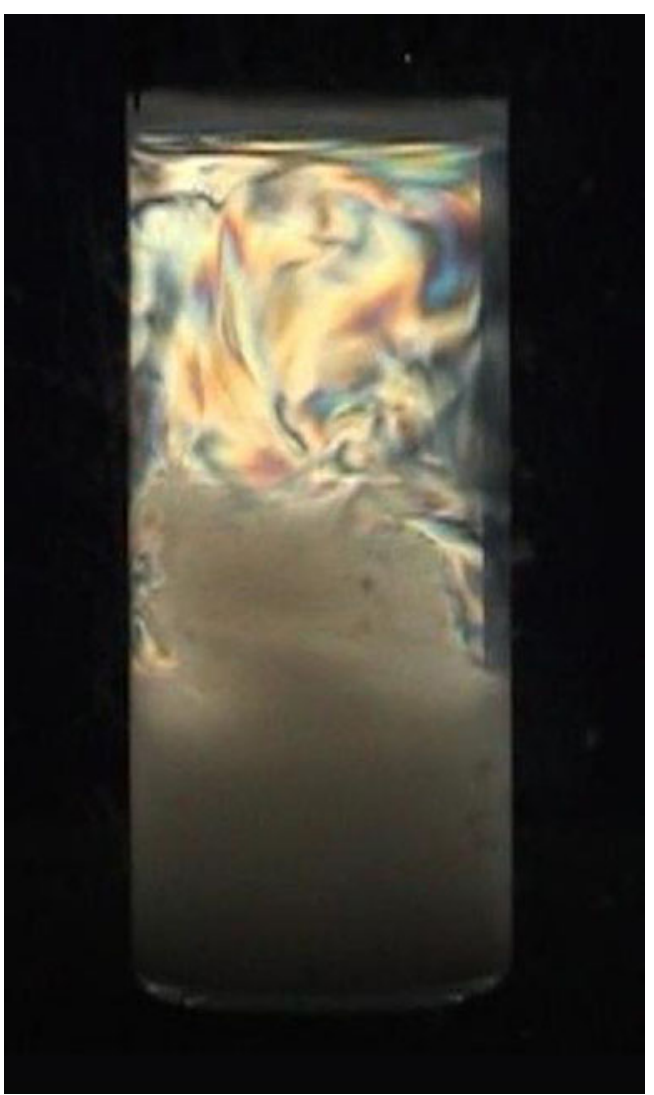

Fig. 2 Example of flow birefringence in a colloidal dispersion of clay particles (Credits Elisabeth Lindbo Hansen, Norwegian University of Science and Technology). A detailed description is given in the text

\section{The Dust Side of the Force Team}

The Dust Side of the Force team is composed of five German students from the Institute of Planetology of the University of Muenster in Muenster (Germany). The aim of their experiment was to quantify the GTEffect and its gravitational dependency.

In a low pressure gaseous environment the GTEffect can be established in translucent and dusty bodies through illumination (Wurm and Krauss 2006). It is an interplay of two basic concepts: a solid-state greenhouse effect $(G)$ and a temperature gradient $(T)$.

If the resulting GT-force, $F_{\mathrm{GT}}$, overcomes the gravity $\mathrm{Fg}$ and the cohesion Fco between the single dust particles, dust is ejected from a body's surface or a dust bed. Figure 4 shows an example of dust ejection from a dust bed due to the GT-effect.

The aim of this student experiment was to determine the intensity threshold for particle ejection caused by the GT-effect. By illuminating, through a high power halogen lamp, a dust sample (JSC Mars 1A soil analogue) placed in a vacuum chamber with residual 
Fig. 3 Sketch of the Complex team's experimental setup (Credits Henrik Hemmen, Norwegian University of Science and Technology). A detailed description is given in the text

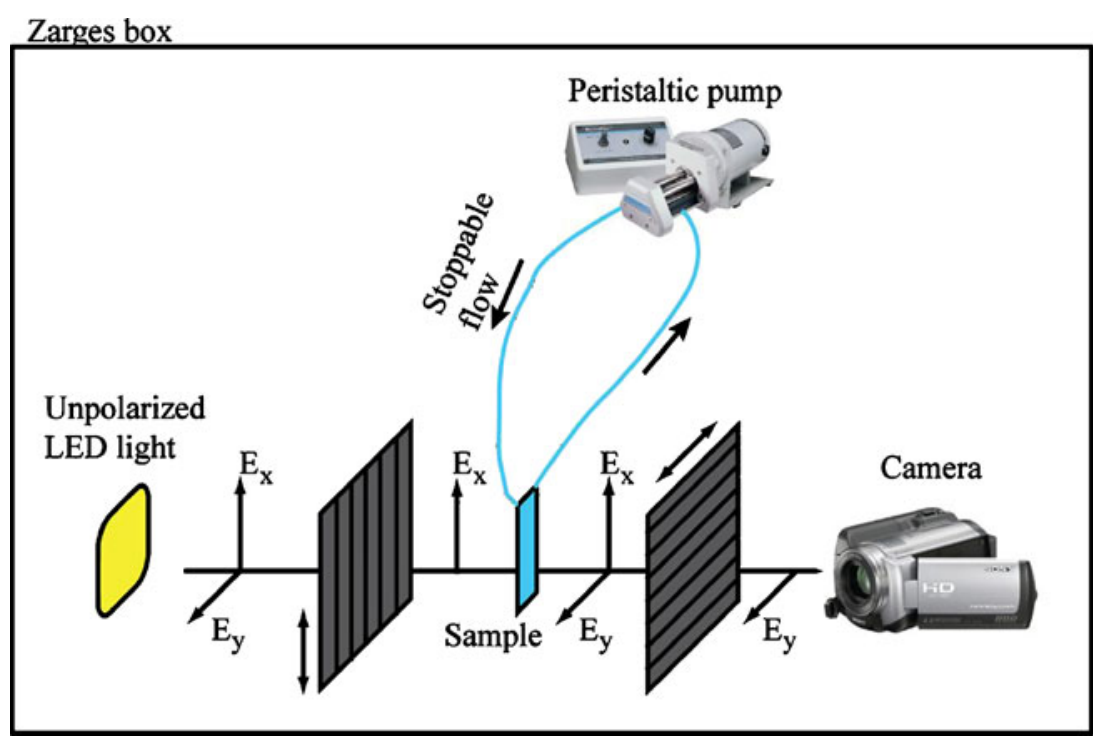

pressure of a few mbar, a temperature gradient was created inside the dust sample. The interaction of the dust particles and the gas results in a dust lifting force (see Fig. 5 for the experimental setup). The students measured intensity thresholds for the eruptions in all three gravity phases (microgavity, hypergravity and Earth's gravity) at different pressures in the order of a few mbar.

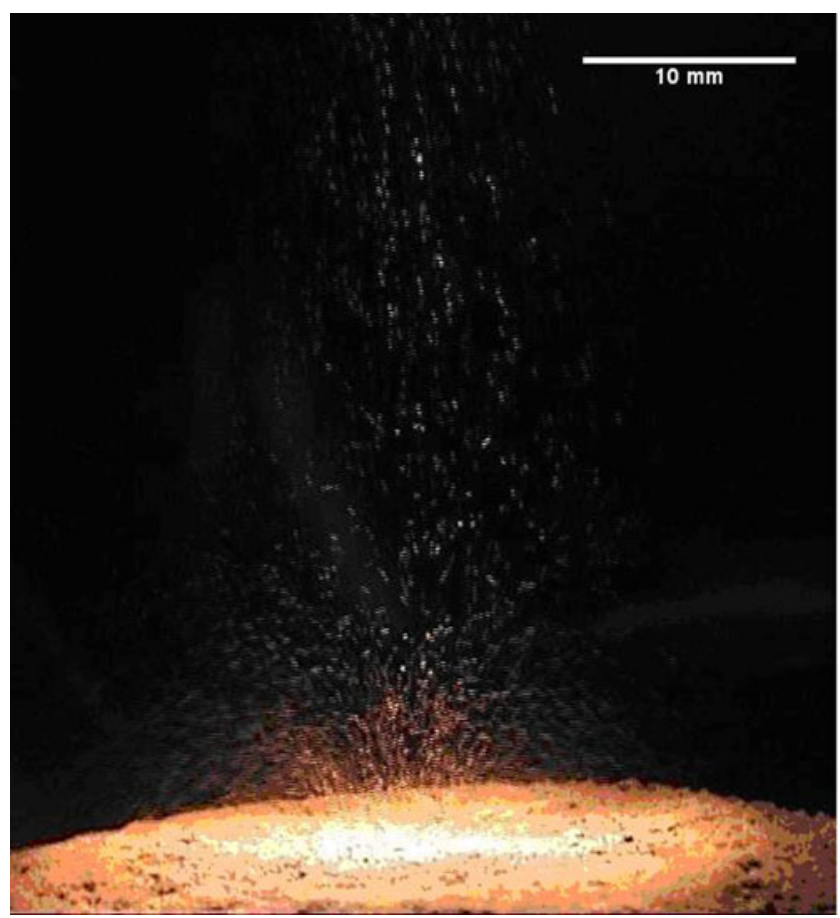

Fig. 4 Example of dust ejection from a dust bed due to the GTeffect (Credits The Dust Side of the Force team, University of Muenster). A detailed description is given in the text
As the GT-effect is thought to be important in planet formation and formation of dust devils and storms on Mars, this successful student experiment may contribute in the future to a better understanding of these phenomena.

\section{The AstEx Team}

The AstEx team is composed of two British students from the Open University in Milton Keynes (UK) and the University of Nice Sophia Antipolis in Nice (France). The aim of their experiment was to study the behaviour of granular material under shear stress to better understand the surface geology of small bodies in our solar system.

The students' experiment investigated:

- how a steady state flow is achieved in a granular material in microgravity conditions

- how a steady state flow in microgravity differs to a steady state flow on Earth

- what effect reversing the direction of shear has on the steady state flow already initiated in microgravity

The experiment was conducted using a TaylorCouette shear cell modified for microgravity conditions as illustrated in Fig. 6.

The outer cylinder was fixed in place but the inner cylinder was allowed to rotate and was connected to the motor. The bottom floor between the two cylinders was smooth and fixed in place. The gap between the two cylinders was filled with spherical glass beads of diameter $d$, such that the gap has a width of $\sim 50 d$ (see 
Fig. 5 Picture of the Dust Side of the Force team's experiment rack (Credits The Dust Side of the Force team, University of Muenster). A detailed description is given in the text

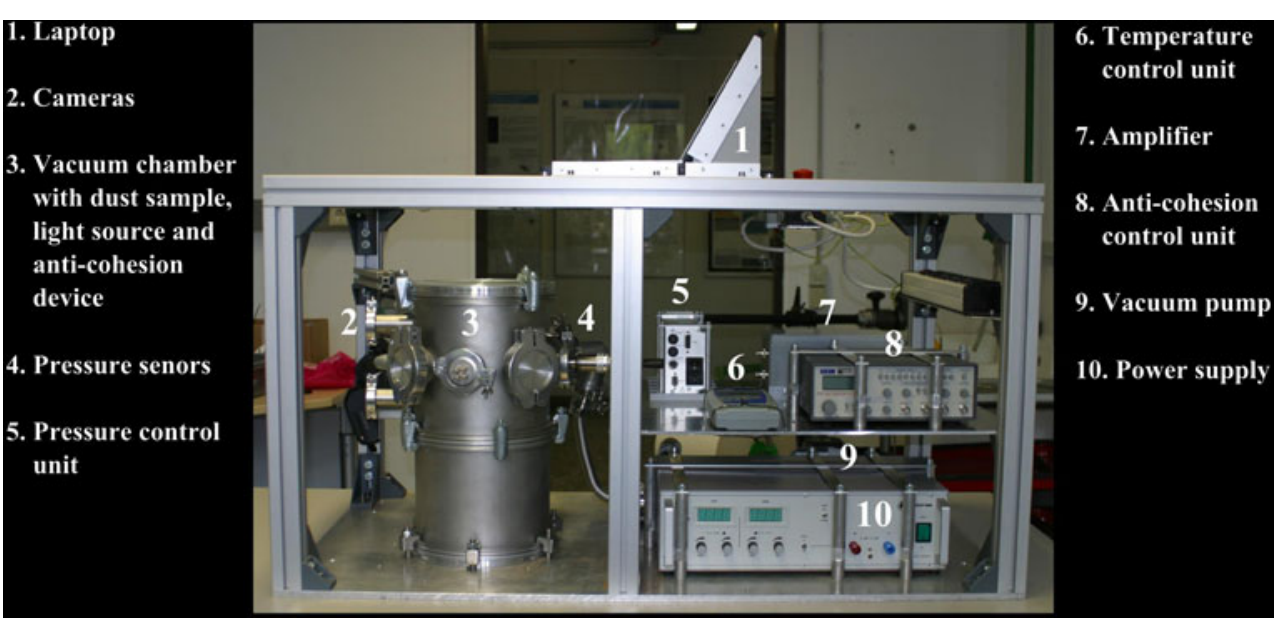

Fig. 7). Glass beads of the same diameter were glued to both cylinder surfaces.

A granular flow was started by applying rotational shear forces to the granular material. Individual glass beads were tracked by two high speed cameras so that their positions and velocities could be determined.

The three investigations were repeated with glass beads of different sizes $(2-4 \mathrm{~mm})$, and with different shear rates $(\max 1 \mathrm{rpm})$ to determine what effect these properties have (Rozitis et al. 2009).

A better understanding of granular material dynamics under different conditions would lead to a wide range of applications. Results from this successful student experiment may, for example, help to understand the nature of asteroid surfaces and to design a suitable sampling mechanism for asteroid sample return missions. Due to their small size and very low mass,

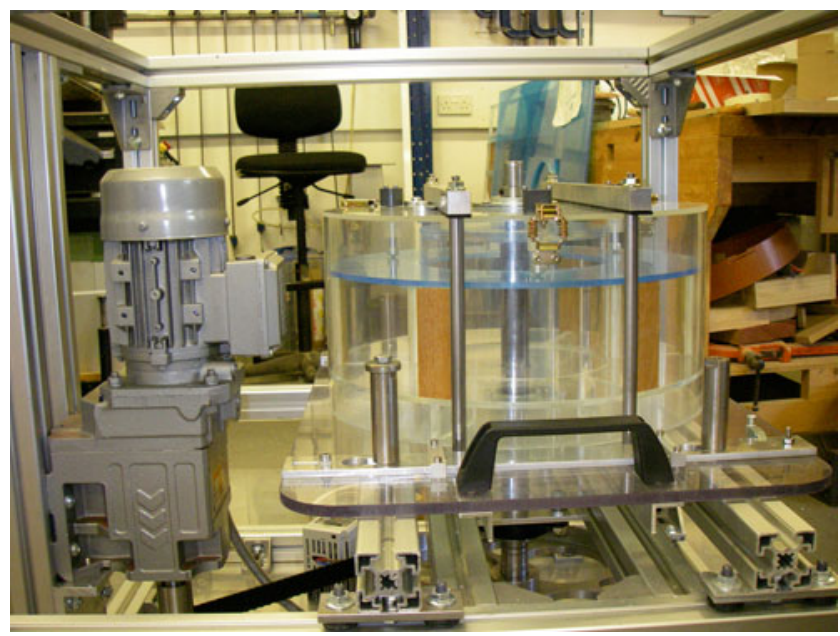

Fig. 6 Picture of the AstEx team's experiment rack (Credits The AstEx team, The Open University and the University of Nice Sophia Antipolis). A detailed description is given in the text asteroids have microgravity conditions at their surface. Despite having a microgravity surface, asteroids can still have granular material residing on their surface.

\section{The ABCtr MicroG Team}

The ABCtr MicroG team is composed of four Spanish students from the Fundació CIM of the Polytechnic University of Catalonia and the Autonomous University of Barcelona in Barcelona (Spain). They investigated the behaviour of particular biological agents called $\mathrm{ABC}$ transporters in microgravity conditions. $\mathrm{ABC}$ transporters are membrane enzymes present in all cells of the human body. Their main function is to act as cell depurators, cleaning toxic waste and drugs from the body as sketched in Fig. 8 (Galvinas et al. 2004).

The student experiment aimed to determine the activity of an ABC transport model (MRP2) while transporting a model substrate (estradiol glucuronide) in microgravity and to compare its performance with equal terrestrial controls to ascertain if their function can be altered by microgravity.

As illustrated in Fig. 9, they used four different automatic units in parallel to determine the active drug modulation capacity and the exact phase where microgravity may affect transport activity.

ABC transporters have been obtained using biotechnology and genetic engineering techniques. The transporters were placed on vesicle shaped structures able of capturing a given drug from a medium. The reaction was triggered by ATP (adenosine tri-phosphate) which provided chemical energy for drug transportation. Drug-loaded vesicles were recovered by filtration and by gas chromatography-mass spectrometry detection systems and the drug quantity was measured.

Results from this successful student experiment may shed more light on $\mathrm{ABC}$ transporters themselves as a 
Fig. 7 Sketch of a Taylor-Couette cell (Toiya 2006; Credits The AstEx team, The Open University and the University of Nice Sophia Antipolis)

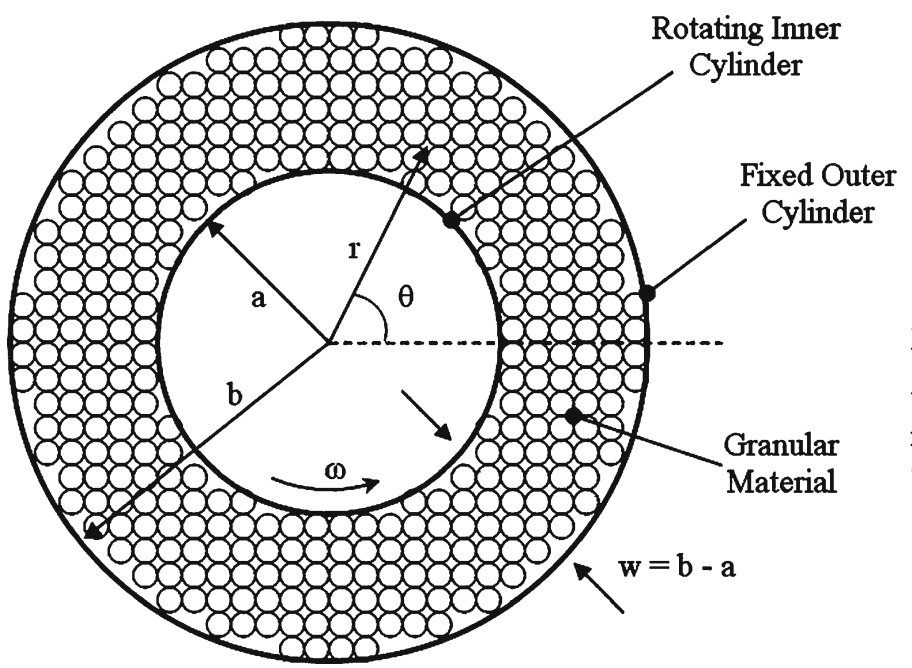

a - Inner Cylinder Radius

b - Outer Cylinder Radius w - Width of Shear Region $r$ - Radial Distance $\theta$ - Angular Distance $\omega$ - Inner Cylinder Rotation Rate complex system, and this system's role in space and terrestrial pharmacology, thereby eventually helping to improve terrestrial cancer treatments and fostering space drug research for future Moon and Mars missions.

"Fly Your Thesis!" 2010

A second edition of the FYT programme was launched in April 2009 and a preliminary selection of 12 student teams was announced at the beginning of September 2009:

- GRAPPA: Gravitationless accretion of protoplanetary bodies and rubble pile asteroids, University of Leicester, Leicester (UK)
- ARID: Electrostatic self-assembly of topsoil particles to study the reorientation of water repellent organic coatings, University of Amsterdam, FNWI-IBED-Earth Sciences, Amsterdam (The Netherlands)

- QNEM \& nano's on board!: Is nano's thermal diffusivity different in micro-g?, Université Libre de Bruxelles, Microgravity Research Center, Brussels (Belgium)

- TREX Parabolic Team: Investigation of the effectiveness of "Einstein's teacup" phenomenon in microgravity using parabolic flights, Luleå University of Technology, Kiruna (Sweden)

- Lark: Influence of gravity on the effects of anaesthetic materials in mouse model utilizing heart rate variability measurement and analysis methods,
Fig. 8 Sketch of the cell depurator function of $\mathrm{ABC}$ transporters (Credits The ABCtr MicroG team, Fundació CIM). A detailed description is given in the text
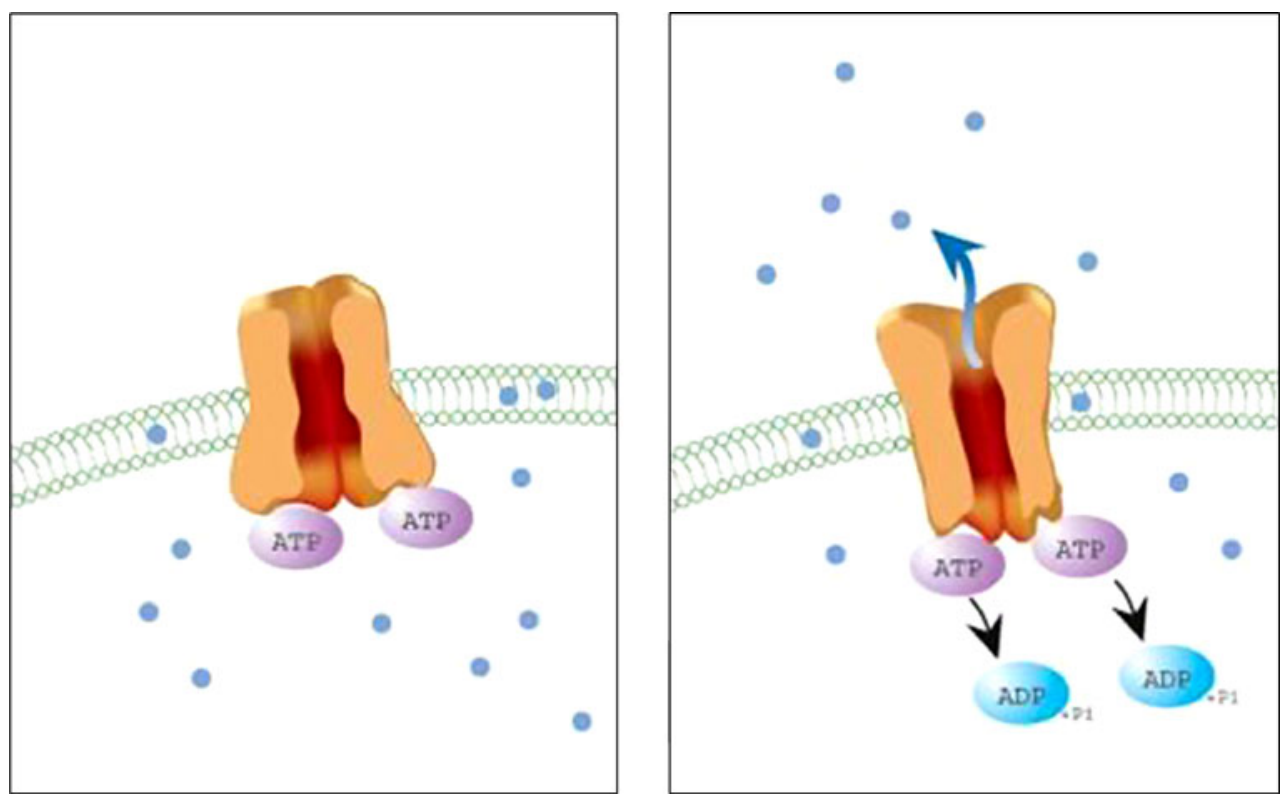
Fig. 9 Sketch of the experiment setup of the ABCtr MicroG team (Credits The ABCtr MicroG team, Fundació CIM). A detailed description is given in the text

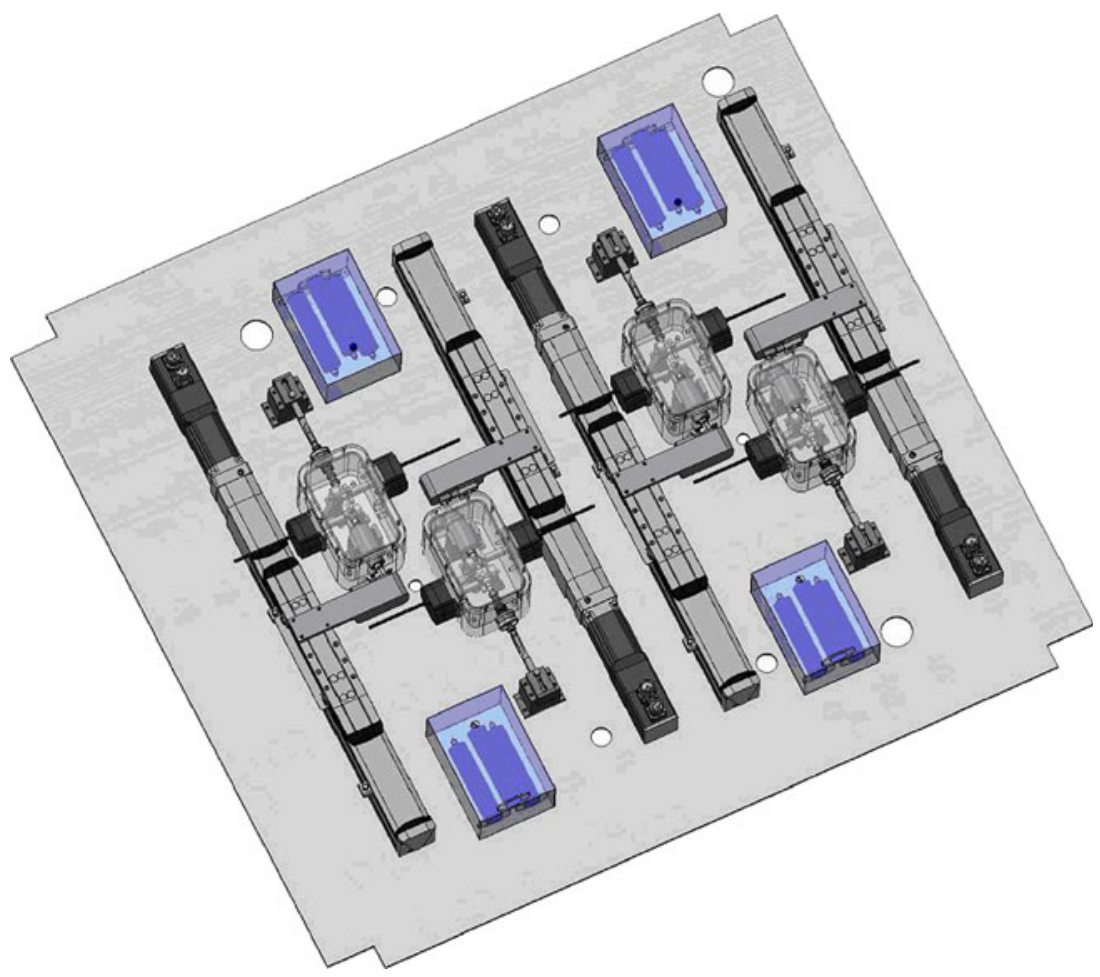

Szent Istvan University, Faculty of Veterinary Science, Budapest (Hungary)

- GSI: Effect of microgravity and flight-related stress on radio-sensitivity, Technical University of Darmstadt, Darmstadt (Germany)

- Team Chaos: Chaotic Dripping in Reduced Gravity, Kingston University, Faculty of Engineering, Kingston (UK)

- Sparky Space Cells: Investigation of proposed porous and micro channel material electrodes in a low temperature proton exchange membrane fuel cell for Earth and space applications, Dublin City University, Mechanical Engineering Department, Dublin (Ireland)

- MuSpace2010: Analysis of metabolic and physical behaviour of muscle models in gravityaltered environments, CRIM Lab, Scuola Superiore Sant'Anna, Pisa (Italy)

- KUL ADACS: Low Cost Attitude determination and control system, Katholieke Universiteit Leuven, Mechanical Engineering, Leuven (Belgium)

- CMEFE: Micro-g experiment for liquid management device, CMEFE, Petit Lancy-GE (Switzerland)

- Supermassive B., DEEP IMPACT, Université Joseph Fourier, Grenoble (France).

The final selection of four student teams was announced in January 2010. The GRAPPA, ARID,
QNEM \& nano's on board! and Supermassive B teams will have the opportunity to fly their experiments during an ESA Microgravity Research Campaign beginning of 2011.

\section{Additional ESA Hands-on Activities}

The ESA Education office was endeavouring to extend the range of hands-on activities by developing student programmes for other microgravity and hypergravity ground-based platforms. In 2009, two new hands-on activities called "Drop Your Thesis!" (DYT) and "Spin Your Thesis!" (SYT) were launched. They give European university students access to the Zarm Drop Tower facility in Bremen (Germany) and the ESA Large Diameter Centrifuge facility in Noordwijk (The Netherlands).

\section{“Drop Your Thesis!” (DYT)}

In 2009, a pre-selected student team for the first edition of the FYT programme was offered the opportunity to have its experiment performed in the ZARM Drop Tower facility (http://www.zarm.uni-bremen.de). As illustrated in Fig. 10, this facility is composed of a $146 \mathrm{~m}$ tall steel tube, designed as a vacuum unit, which houses all technical parts to handle the drop capsule. Below the 


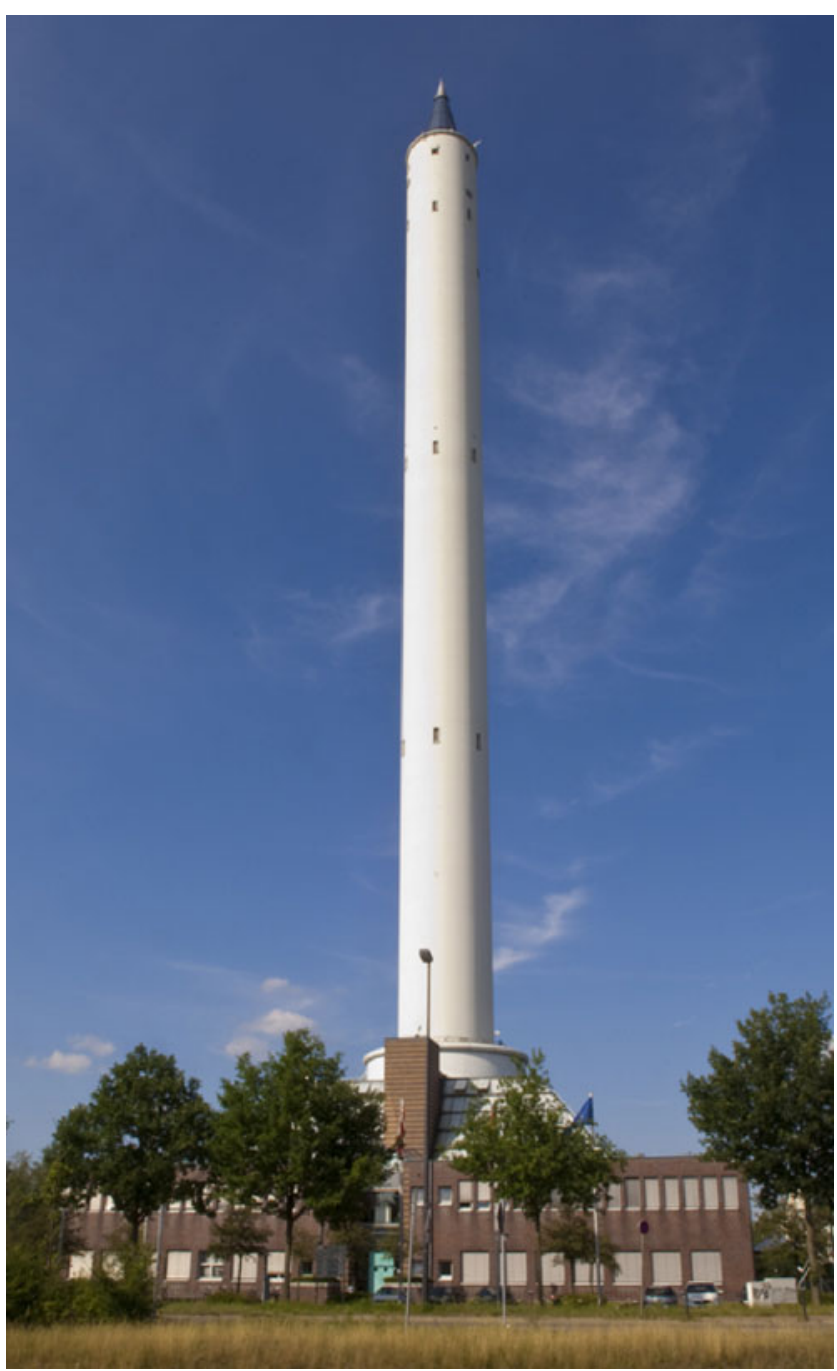

Fig. 10 Picture of the Zarm Drop Tower in Bremen, Germany (Credits ZARM FAB). A detailed description is given in the text

tower, a chamber of $11 \mathrm{~m}$ depth contains the catapult system. The installation delivers $4.74 \mathrm{~s}$ of microgravity in dropping mode and $9.3 \mathrm{~s}$ in the catapulting mode (von Kampen et al. 2006).

The Physic's Addicted team is composed of four students form the University of Porto, in Porto (Portugal). The aim of their experiment was to study the influence of microgravity on polymer dispersed liquid crystal properties to optimise polymerisation processes on Earth. This first student campaign took place in November 2009. The student team catapulted successfully three times their experiment.

In parallel to this first student campaign, ESA Education Office developed the "Drop Your Thesis!" programme. The objective of this educational activity is to give one university student team each year the op-

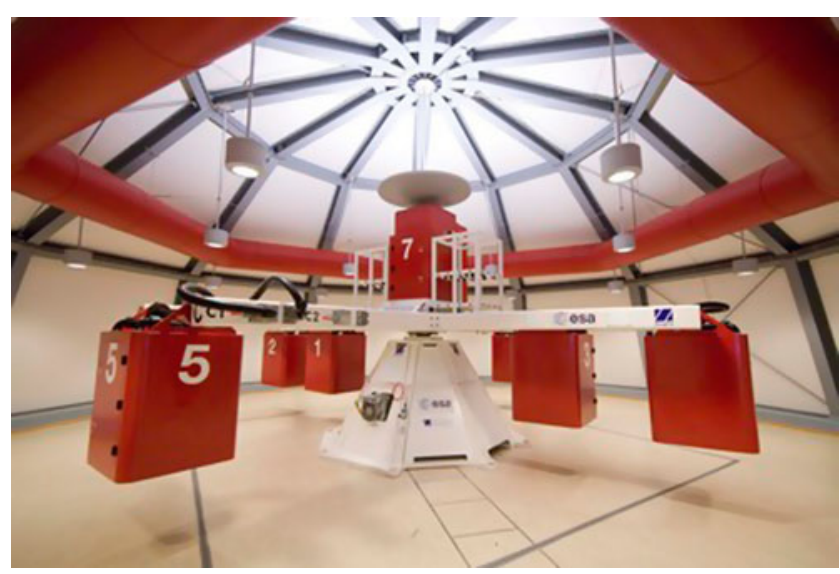

Fig. 11 Picture of the ESA Large Diameter Centrifuge in Noordwijk, The Netherlands (Credits ESA). A detailed description is given in the text

portunity to drop a few times a microgravity scientific experiment in the ZARM Drop Tower, as part of their Masters or Ph.D. thesis. The first call was launched in November 2009 and a first campaign will be held one year later.

It is currently being assessed whether the opportunities offered could be extended to include the INTA Drop Tower facility in Madrid, Spain.

\section{"Spin Your Thesis!” (SYT)}

The ESA Education Office also prepared a first call of opportunities to perform experiments in the ESA Large Diameter Centrifuge (van Loon et al. 2008). This centrifuge has a diameter of eight meters and is composed of four arms which can support up to six gondolas (see Fig. 11). In order to perform the experiments, the acceleration of the facility varies from 1 to 20 times Earth's gravity within at least $60 \mathrm{~s}$.

The "Spin Your Thesis!" programme will permit European university students, to design, build and perform hypergravity experiment as part of their syllabus. The objective is to have a two week campaign with four student experiments. The first call of opportunities was made beginning of November 2009 and first campaign should take place in spring or summer 2010.

\section{Summary}

Since ESA hands-on space project activities began in the mid-1990s with the first student parabolic flight 
campaign to present day activities it is estimated that over 3,600 students distributed over 22 ESA Member and Cooperating States have benefited from it.

With the three new gravity-related ESA Education Office programmes, "Fly Your Thesis!", "Drop Your Thesis!" and "Spin Your Thesis!" more then 8 student teams will have, each year, the unique opportunity to design, build, and eventually perform, in microgravity or hypergravity, a scientific experiment which is linked to their syllabus.

Acknowledgements We would like to thank the following for their support in the "Fly Your Thesis!" programme:

- the ESA Utilisation Department of the Human Space Flight Directorate;

- The European Low Gravity Research Association, ELGRA and the individual mentors for these programmes;

- Novespace the company in charge of the Airbus A300 "Zero-G";

- Zarm FAB the company in charge of the Zarm Drop Tower.

The following student teams, selected for "Fly Your Thesis!" 2009 programme, have contributed to this manuscript and we would like to thank them for their inputs:

- The Complex team from the Norwegian University of Science and Technology in Trondheim (Norway);

- The Dust Side of the Force team from the Institute of Planetology of the University of Muenster, in Muenster (Germany);

- The AstEx team from the Open University in Milton Keynes (UK) and the University of Nice Sophia Antipolis in Nice (France);

- The ABCtr MicroG team from the Fundació CIM of the Polytechnic University of Catalonia in Barcelona (Spain).

Open Access This article is distributed under the terms of the Creative Commons Attribution Noncommercial License which permits any noncommercial use, distribution, and reproduction in any medium, provided the original author(s) and source are credited.

\section{References}

Callens, N., Ventura-Traveset, J., de Lophem, T.-L., Lopez de Echazarreta, C., Pletser, V., van Loon, J.J.W.A.: Fly Your Thesis! - an astronaut experience-ESA Parabolic Flights opportunities for university students in collaboration with ELGRA. ELGRA News 26, 81. ELGRA Biennial Symposium, Bonn, Germany (2009)

Emma, F., Ventura-Traveset, J., Walker, R., Page, H., Lopez de Echazarreta, C., de Lophem, T.-L., Cross, M., Berglund, P., Nikolaidis, V.: Paper IAC-08-E1.1.2, "ESA Hands-on projects education strategy". In: 59th International Astronautical Federation Congress, Glasgow, Scotland (2008)

Galvinas, H., Krajcsi, P., Cserepes, J., Sarkadi, B.: Curr. Drug Delivery 1, 27-42 (2004)

Okubo, T., Tsuchida, A., Yoshimi, H., Taguchi, K., Kiriyama, S.: Colloid. Polym. Sci. 280, 228-233 (2002)

Pletser, V., Gharib, T., Gai, F., Mora, C., Rosier, P.: The 50 parabolic flight campaigns of the European Space Agency to conduct short duration microgravity research experimentation. In: Paper IAC-09-A2.5.1, 60th International Astronautical federation Congress, Daejeon, Korea (2009)

Rozitis, B., Murdoch, N., Green, S.F., de Lophem, T.-L., Michel, P.: AstEx microgravity experiment: simulating asteroid regoliths. In: Paper IAC-09.A2.3.9, 60th International Astronautical federation Congress, Daejeon, Korea (2009)

Toiya, M.: Onset of granular flows by local and global forcing. Available at: http://hdl.handle.net/1903/3886 (2006)

van Loon, J.J.W.A., Krause, J., Cunha, H., Goncalves, J., Almeida, H., Schiller, P.: The Large Diameter Centrifuge, LDC, for life and physical sciences and technology. In: Proc. of the Life in Space for Life on Earth Symposium, Angers, France (2008). (ESA SP-663, December 2008)

von Kampen, P., Kaczmarczik, U., Rath, H.J.: The new Drop Tower catapult system. Acta Astronautica. 59(1-5), 278-283 (2006)

Wurm, G., Krauss, O.: Phys. Rev. Lett. 96, 134301 (2006). doi:10.1103, Phys. Rev. Lett. 96.134301 